\title{
Gut microbiota: a potential new territory for drug targeting
}

\author{
By: Wei Jia, Houkai Li, Liping Zhao and Jeremy K. Nicholson
}

Jia, W., Li, H., Zhao, L., Nicholson, J.K. (2008) Gut microbiota: a potential new territory for drug targeting, Nature Reviews Drug Discovery. 7: 123-131. DOI10.1038/nrd2505

Made available courtesy of Nature Publishing Group: http://npg.nature.com

***Note: Figures may be missing from this format of the document

\begin{abstract}
:
The significant involvement of the gut microbiota in human health and disease suggests that manipulation of commensal microbial composition through combinations of antibiotics, probiotics and prebiotics could be a novel therapeutic approach. A systems perspective is needed to help understand the complex host- bacteria interactions and their association with pathophysiological phenotypes so that alterations in the composition of the gut microbiota in disease states can be reversed. In this article, we describe the therapeutic rationale and potential for targeting the gut microbiota, and discuss strategies and systems-oriented technologies for achieving this goal.
\end{abstract}

\section{Article:}

Most current searches for new drugs are focused on identifying a pharmacologically effective agent that specifically interacts with one molecular target in the eukaryotic host cell to regulate biochemical alterations in a diseased state and reinstate healthy- state biochemistry. During the past 30 years, such an approach has generated highly successful medicines. However, it does not completely exploit the complex regulatory network that has been engineered through evolutionary processes in humans, who could be viewed as 'superorganisms' with an indispensable internal ecosystem: the gut microbiota ${ }^{1}$.

The human gastrointestinal (GI) tract is home to a complex consortia of trillions (approximately $1 \times 10^{13}$ to $1 \times 10^{14}$ ) of microbes, thousands of bacterial phylotypes, as well as hydrogen-consuming methanogenic archaea, colonizing the entire length of the gut with a collective genome (also termed as microbiome) that contains at least 100-times as many genes as our own genome ${ }^{2}$. Although largely unexplored and under-appreciated, our gut microbiota plays an intricate and sometimes pivotal role for our health and well-being. Emerging 'omics' technologies, such as metagenomics and metabonomics, are now being applied to the study of the gut microbial ecology at the molecular level, generating new insights and opportunities to reveal the medical functions of the gut microbiota to human health ${ }^{2-4}$.

Accumulating evidence indicates that the gut microbiota is instrumental in energy metabolism and immune function of the host, and has a crucial role in the development of numerous conditions including obesity $y^{5,6}$, diabetes ${ }^{7}$, non-alcoholic fatty liver disease ${ }^{4}$, inflammatory bowel diseases ${ }^{8}$, and even cancers ${ }^{9}$. Unlike the human genome, which is intact and rarely manipulated by xenobiotic intervention, the composition of commensal gut microbiota is readily changeable. The plasticity of the microbiome may allow a specific or systematic manipulation of certain gut 
microbiota associated with host diseases. Therefore, the exploration of the gut microbiota might not only revolutionize therapeutic strategies for many diseases, but also improve the productivity of the pharmaceutical industry, which has recently made huge investments in the discovery of novel targets and drug candidates with limited success so far. With this in mind, this article provides perspectives for the therapeutic and prophylactic management of diseases and disorders that involve the gut microbiota, and suggests strategies for gut microbiota-oriented drug discovery.

\section{A microbial organ}

The human intestine is more densely populated with microorganisms than any other organ, and is a site where they exert strong influences on human biology. With this in mind, the entire system of the human gut microbiota can be pictured as a 'microbial organ' within the intestine, which contributes to diverse mammalian processes including protective functions against pathogens and immune-system modulation, the metabolic function of fermenting non- digestible dietary fibre, and the anaerobic metabolism of peptides and proteins that results in the recovery of metabolic energy for the host ${ }^{10-12}$.

The intestinal mucosa is the main interface between the immune system and the external environment and therefore has an important role in the host-flora interaction. The commensal microbiota profoundly influences the development of humoral components of the gut mucosal immune system ${ }^{12}$, acting as a crucial factor in the prevention of exogenous pathogen intrusion. Metabolites, including digested dietary nutrients, are absorbed by the gut, and together with various non-nutrient compounds produced by the microbiota, are co-metabolized by host enzymes such as the cytochrome P450 and conjugating enzymes in the liver ${ }^{13}$. The resulting metabolites that are derived from both host and microbial processes are then returned to the gut by the bile and possibly other secretory routes for further metabolism and/or excretion. On one hand, the daily metabolism of food involving the different types of metabolic substrates and the secretion of peptic acid, bile and pancreatic fluids regulates the composition of the intestinal mucosa by hindering the colonization of the stomach and proximal small intestine by most bacteria, and changing the composition of enteric bacteria in the distal small intestine and the large intestine. On the other hand, commensal-derived metabolites and the composition of bacterial surface structures can have various effects on the host immune system ${ }^{14}$. Meanwhile, commensal bacteria can modulate gene expression in the host to create a suitable environment for themselves and prevent the growth of other competitive bacteria within the ecosystem ${ }^{11}$. 


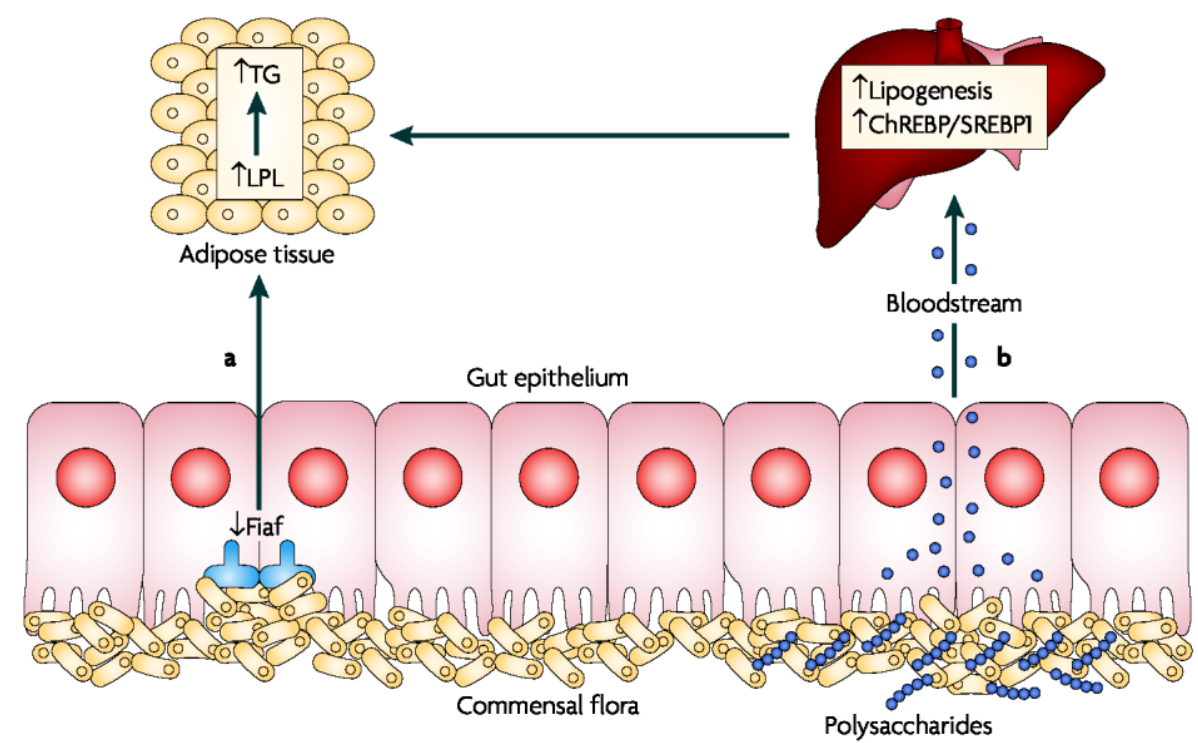

Figure 1 | Schematic view of how the gut microbiota affects host fat storage. The gut microbiota increases lipoprotein lipase (LPL) activity in adipocytes to promote storage of calories harvested from the diet into fat through two mechanisms, as discovered by Gordon and colleagues ${ }^{17}$. In the first mechanism, the gut microbiota selectively suppresses fasting-induced adipocyte factor (FIAF, also known as ANGPTL4), a member of the angiopoietin-like family of proteins, leading to the upregulation of $L P L$ (a). In the second mechanism, the gut microbiota metabolize the non-digestible polysaccharides and induce de novo hepatic lipogenesis through the absorption of resulting monosaccharides (b). ChREBP, carbohydrate response element binding protein; SREBP1, sterol regulatoy element binding protein 1; TG, triglyceride.

Being such a microbial organ, our gut flora not only plays a significant role in disease onset and progression, but also influences drug bioavailability and toxicological incidence via microbialdependent drug metabolism. For example, the metabolism of ginsenosides, the principal bioactive components in Radix Ginseng. Ginsenoside Rb1, a saponin of protopanaxadiol, is metabolized by $\beta$-D-glucosidases in the gut microbiota into $20-O-\beta$-D-glucopyranosyl-20(S)protopanaxadiol, which exhibits significant antitumour and anti-allergic activities ${ }^{15}$. A toxicological research programme, the Consortium on Metabonomic Toxicology, has confirmed that the unusual metabolic profiles observed in a small proportion of the experimental rats treated with model toxic compounds are attributed to different gut microbiome contributions 16 , suggesting that variations in drug metabolism under identical treatment are in direct association with the inter-subject diversity of the gut microbiota.

A dynamic Pachinko model of metabolism, postulated by Nicholson and Wilson, suggests that the outcome of drug metabolism in mammalian transformation systems is not pre-arranged or determined absolutely, but is probabilistic and conditional on the sequence and sites of the previous metabolic events, and strongly influenced by the distribution of key metabolizing enzymes and transporter molecules ${ }^{13}$. It is therefore reasonable for an idiosyncratic toxicity to arise in selected individuals owing to unusual metabolic fates or interactions of drugs that are due to rare, but toxic, metabolic outcomes that become favoured by unusual combinations of genetic and environmental factors. With the same perspective, disease onset at a specific time point and with a unique phenotype among individuals can be considered a result of complex and probabilistic metabolic co-regulation by the host genome and the intrinsic microbiome in response to different dietary and environmental stress factors. 


\section{The gut microbiota and diseases}

Recent publications have shown that the gut microbiota has a profound influence on the onset and progression of important human diseases that are currently lacking therapeutic solutions ${ }^{5,9}$. For example, differences in caloric extraction efficiency from food may be determined by the composition of the microbiota, which, in turn, may contribute to differential body weights ${ }^{5}$. Gut microbial species break down non-digestible polysaccharides and promote absorption of the resulting monosaccharides from the gut lumen, facilitating the induction of de novo hepatic lipogenesis. Additionally, fasting-induced adipocyte factor (FIAF; also known as ANGPTL4), a member of the angiopoietin-like family of proteins, is selectively suppressed in the intestinal epithelium in the presence of enteric bacteria, leading to a microbiota-induced deposition of triglycerides in adipocytes ${ }^{17}$ (FIG. 1). When mice are exposed to a high-fat diet, the gut microbial metabolism affects the conversion of choline into methylamines, reducing the bioavailability of choline to mimic the effect of a choline-deficient diet, and causing nonalcoholic fatty liver disease ${ }^{4}$. These findings raise the possibility of manipulating the microbiotic environment to treat or prevent obesity and associated diseases.

Certain environmental toxins such as dimethylarsine can be metabolized by specific strains of the gut resident Escherichia coli to produce potentially toxic micrometabolites, underlying the carcinogenicity of this type of compound in the gut ${ }^{18}$. Production of toxins, particularly neurotoxins or toxic metabolic products, by such microorganisms might also mediate neurological disruptions. We anticipate that a number of neurological disorders, such as attention-deficitlhyperactivity disorder (ADHD) and Tourette syndrome, which may be associated with this type of pathogenesis ${ }^{19,20}$, will eventually find treatment solutions in the gut microbiota.

It is widely speculated, although controversial, that perturbations in the GI micro- biota composition as a result of the wide use of antibiotics in modernized countries may be linked to several conditions including obesity, insulin resistance, diabetes, irritable bowel syndrome and diarrhoea $^{21-23}$. The mechanism behind this speculation is that in normal conditions, the stability of the microbial structure is maintained by the equilibrium of resident bacteria. However, this may be readily disrupted by antibiotics, leading to the overgrowth of antibiotic-resistant pathogens that are associated with numerous complex disorders, such as toxigenic Clostridium difficile-associated pseudomembranous colitis ${ }^{24}$. Additionally, antibiotics can have a long-term impact on the microbial ecology in our gut; a recent study shows that a short- term antibiotic exposure can have persistent consequences for individuals for up to 2 years post-treatment ${ }^{25}$.

Taken together, it is apparent that changes in the Western lifestyle, together with the abuse of antibiotics in our body and our food chain, induce changes in the gut microbial ecology, such changes are likely to affect the genomic and metabolic predisposition of the host towards different energy storage 


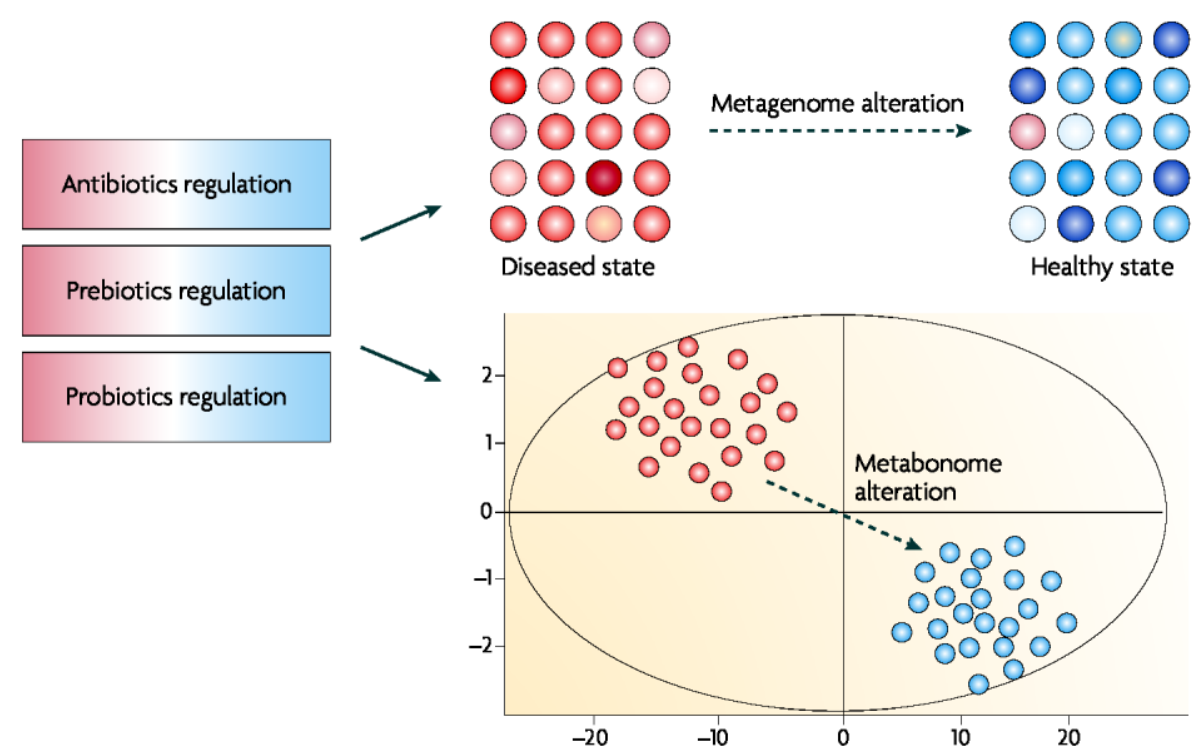

Figure 2| Schematic view of gut microbiota-targeted therapeutic strategy. Future therapeutic strategies for complex diseases will include the treatment of the gut microbiome, that is, 'drug the extended genome' strategy. The human microbiome may possess many potential targets, a number greater than the 3,000 targets from the human genome. As the gut microbiota affects metabolic phenotypes and enzyme induction states, which ultimately affects the outcome of interventions, the microbiome can be manipulated to alter interventional outcomes. Combinations of antibiotics, probiotics and prebiotics could be used in a gut microbiota-targeted therapy regimen to regulate the microbiome and, consequently, restore the homeostasis of gut ecology in the host. Such a therapeutic approach can be monitored and evaluated using 'omics' platform technologies, such as metagenomics and metabonomics, by capturing the holistic and dynamic biochemical variations associated with pathophysiological conditions of the host. The integration of metagenomic and metabonomic data into pharmacological and clinical results should be fulfilled to obtain comprehensive diagnostic and prognostic knowledge regarding the complex diseases.

capabilities, metabolic and immune regulatory networks, and pathologies. The gut microbiota could therefore be a previously under-appreciated source of drug targets for the treatment of metabolic disorders, various conditions in the GI tract and also neurological disorders.

\section{The gut microbiota as drug targets}

The knowledge and experience obtained from the management of chronic peptic ulcers induced by Helicobacter pylori suggests that certain chronic diseases are derived from the growth of particular microorganisms that are not inhibited by conventional biochemical modifications in eukaryotic host cells ${ }^{26}$, such microorganisms can be causative agents of disease and are therefore therapeutic targets, which necessitate modification with antibiotics and/or nutritional intervention as a realistic means for disease management.

As we only possess limited knowledge of our gut microbiota and lack most of the molecular details of host-flora interactions, it may be too early to propose a clear microbiota-based drug discovery strategy or therapeutic modality. However, initial considerations of target candidates are expected to centre on those indigenous bacterial species with known genome sequences and biochemical functions, for example, Bacteroides thetaiotaomicron is a Gram-negative anaerobe and a dominant member of the normal distal intestinal microbiota, which devotes a large proportion of its genome to metabolic processes that benefit the host and other commensal 
species $^{27}$. It has an elaborate apparatus for acquiring and hydrolysing otherwise indigestible dietary polysaccharides and influences the transport and regulation of mammalian peroxisome proliferator-activated receptor- $\gamma$ (PPAR $\gamma)$, an intranuclear receptor that is highly expressed in host adipose tissue and involved in the mechanism of human insulin resistance ${ }^{28}$. Certain pathogenic microbial species, which differ distinctly from healthy populations as a result of xenobiotic intervention and pathological development, could be identified as targets of interest for relevant treatment strategies.

Compositional structures or patterns of the gut microbiota that are associated with certain diseases constitute a new type of drug target. such a concept evolves from recent work comparing the distal gut microbiota of genetically obese mice and their lean littermates, as well as those of obese and lean human volunteers, in which obesity was found to be associated with changes in the relative abundance of the two dominant bacterial divisions, the Bacteroidetes and the Firmicutes, and a dominant archaeon, Methanobrevibacter smithii ${ }^{5,6,29}$. Recent comparative genomic profiling analysis has revealed a wide distribution of the type III secretion system in commensal bacteria and pathogens ${ }^{30}$, which is not only a specific outcome of host cell infection, but also an indication of interactive communication between commensal bacteria and the host. Therefore, we speculate that different compositional structures of the gut microbiota in association with certain diseases may use different secretion patterns or modes to communicate with host cells and participate in the host metabolic network. These different secretion patterns may produce a characteristic secretome or small-molecule metabonome that could be measured by biochemical analysis of urinary and faecal samples. further studies are expected to determine whether commensal bacteria with certain compositional structures will mediate the host immune surveillance in the gut by means of specific secretion patterns. Moreover, the 'omics' sciences such as metabonomics and proteomics can be utilized to monitor the subtle variation resulting from different compositional structures of commensal bacteria under certain pathological conditions.

\section{Gut microbiota-targeted therapies}

We envisage that two strategies may be taken for the development of future gut microbiotatargeted therapies. The first is the direct elimination or modification of a well-defined, specific gut microorganism, or certain species of bacteria, as disease target(s), in a similar way to the treatment of chronic peptic ulcer induced by H. pylori using antibiotics ${ }^{31}$. An alternative method for such a strategy is vaccination using an antigenic epitope of a bacterium or toxin of interest to elicit an immune response capable of interacting with the gut flora upon microbe proliferation in the gut. However, limitations of these methods may include the lack of specificity of the broad-spectrum antibiotics and the vaccination systems available, which will result in unexpected destruction of the beneficial microbial community and a long-term disturbance of gut ecology. Additionally, the frequent exposure of the gut microbiota to antibiotics or vaccines 


\begin{tabular}{|c|c|c|c|}
\hline $\begin{array}{l}\text { Disease or } \\
\text { disorder }\end{array}$ & $\begin{array}{l}\text { Association of gut microbiota } \\
\text { with disease }\end{array}$ & $\begin{array}{l}\text { Evidence of gut microbiota- } \\
\text { targeted therapy }\end{array}$ & Refs \\
\hline $\begin{array}{l}\text { Chronic } \\
\text { peptic ulcer }\end{array}$ & $\begin{array}{l}\text { Helicobacter pylori infection } \\
\text { is the pathogenic key to the } \\
\text { development of most chronic } \\
\text { peptic ulcers }\end{array}$ & $\begin{array}{l}\text { Regimen of } H . \text { pylori eradication } \\
\text { with antibiotics and proton-pump } \\
\text { inhibitory agents }\end{array}$ & 26,57 \\
\hline $\begin{array}{l}\text { Antibiotic- } \\
\text { associated } \\
\text { diarrhoea }\end{array}$ & $\begin{array}{l}\text { The suppression of antibiotic- } \\
\text { sensitive bacteria and over- } \\
\text { growth of antibiotic-resistant } \\
\text { species lead to intestinal } \\
\text { dysfunction }\end{array}$ & $\begin{array}{l}\text { Treatment with probiotics, such as } \\
\text { the yeast Saccharomyces boulardii, } \\
\text { together with antibiotics is effective } \\
\text { in the prevention of antibiotic- } \\
\text { associated diarrhoea }\end{array}$ & 58,59 \\
\hline $\begin{array}{l}\text { Ulcerative } \\
\text { colitis }\end{array}$ & $\begin{array}{l}\text { Abnormal immune response } \\
\text { to commensal bacteria, and } \\
\text { increased numbers of intestinal } \\
\text { microorganisms, but reduced } \\
\text { numbers of protective bacteria } \\
\text { such as Lactobacilli and } \\
\text { Bifidobacteria }\end{array}$ & $\begin{array}{l}\text { Short-term benefits were observed } \\
\text { with antibiotic or probiotic/ } \\
\text { synbiotic therapy }\end{array}$ & 42,60 \\
\hline $\begin{array}{l}\text { Crohn's } \\
\text { disease }\end{array}$ & $\begin{array}{l}\text { Inadequate clearance of ingested } \\
\text { microorganisms by dysfunctional } \\
\text { intestinal macrophages } \\
\text { (hypothesized mechanism) }\end{array}$ & $\begin{array}{l}\text { Reinstating the balance of intestinal } \\
\text { microflora with probiotics, } \\
\text { prebiotics and/or antibiotics, such } \\
\text { as the non-absorbable antibiotic } \\
\text { rifaximin }\end{array}$ & 61,62 \\
\hline Obesity & $\begin{array}{l}\text { The relative abundance of the two } \\
\text { predominant bacterial divisions, } \\
\text { the Bacteroidetes and the } \\
\text { Firmicutes, affect the efficiency } \\
\text { of energy harvest from diet }\end{array}$ & $\begin{array}{l}\text { It is suggested that manipulation } \\
\text { of the commensal microbial } \\
\text { composition could be a novel } \\
\text { therapeutic approach for obesity }\end{array}$ & 5,6 \\
\hline Diabetes & $\begin{array}{l}\text { No gut microbiota-related } \\
\text { mechanism is established, } \\
\text { but it appears that diabetes } \\
\text { is associated with the gut } \\
\text { microbiota }\end{array}$ & $\begin{array}{l}\text { Oral administration of probiotics } \\
\text { shows a significant antidiabetic } \\
\text { effect in diabetic models }\end{array}$ & $34-38$ \\
\hline $\begin{array}{l}\text { Colorectal } \\
\text { cancer }\end{array}$ & $\begin{array}{l}\text { Conversion of dietary } \\
\text { procarcinogens into DNA- } \\
\text { damaging agents or generation } \\
\text { of carcinogens by particular } \\
\text { commensal bacteria are thought } \\
\text { to be certain causes of colorectal } \\
\text { cancer }\end{array}$ & $\begin{array}{l}\text { Reduced prevalence of colon } \\
\text { cancer was observed in interleukin } \\
10 \text { knockout mice by probiotic } \\
\text { Lactobacilli administration, and } \\
\text { strong ant itumour activity was } \\
\text { achieved by Bifidobacterium longum } \\
\text { therapy in vivo }\end{array}$ & 63,64 \\
\hline $\begin{array}{l}\text { Idiopathic } \\
\text { parkinsonism }\end{array}$ & $\begin{array}{l}\text { Partial involvement of } \\
\text { microorganisms such as } H \text {. pylori }\end{array}$ & $\begin{array}{l}\text { H. pylori is important in the } \\
\text { aetiology/pathogenesis of } \\
\text { idiopathic parkinsonism and useful } \\
\text { for disease categorization and } \\
\text { subsequent treatment }\end{array}$ & $65-67$ \\
\hline
\end{tabular}

may induce rapid genetic modification of the gut microbiota, which would accelerate the development of resistance to such therapeutic means. A thorough molecular understanding of host-flora interactions and pathologies is a prerequisite for these therapeutic approaches to be effectively conducted with minimal long-term adverse consequences.

The second strategy is to take a holistic approach, similar to that practiced in traditional Chinese medicine, in which various diseases and conditions are categorized into different syndromes based on a patient's clinical manifestations such as pulse, tongue images and information of urine and excrements, before a treatment is applied. In this strategy, different structural patterns of the gut microbiota that are associated with different clinical manifestations are identified and categorized using profiling methods such as metabonomics and metagenomics. The metabolic or 
genomic profiling of the gut microbiota from plasma, urinary or faecal samples could allow a stratification of certain gut microbiota structures in association with disease phenotypes. Combinations of antibiotics, probiotics, prebiotics and perhaps laxatives could be used to manipulate the gut microbiota to achieve a therapeutically effective regimen and, eventually, restore the homeostasis of gut ecology in the host (Fig. 2).

Probiotics are live microorganisms that are frequently used as dietary regulators to influence the gut microbiota composition, and confer beneficial effects on the host when adequately administered. Bacterial constituents or secreted metabolites, such as antimicrobial peptides (bacteriocins), may also contribute to the bioactivities of probiotics ${ }^{32}$. Efficient probiotic strains have been found in different bacterial genera including Bifidobacteria, Lactobacilli, Streptococci and non-pathogenic E. coli, and even nematode parasites. Many probiotic effects, including resistance to infection, improvement of allergic diseases and anti-inflammatory properties, are achieved by modifying the host immune response. For example, the enhancement of phagocytic activity of peripheral blood leukocytes and natural killer cells, and the stimulation of both nonspecific secretory immunoglobulin $\mathrm{A}(\operatorname{Ig} \mathrm{A})$ and a specific antibody response ${ }^{33}$. Recent research in enteric bacteria indicates that the use of probiotics is rapidly advancing from the field of nutrition or dietary supplementation towards therapeutic applications for various conditions. Oral administration of Lactobacillus casei in diabetic KK-Ay mice has been shown to significantly decrease plasma glucose levels and inhibit the production of B-cell specific CD4 ${ }^{+} \mathrm{T}$ cells and cytokines - interferon- $\gamma$ (IFN $\gamma$ ) and interleukin 2 (IL2) - which are leading factors involved in the induction of autoimmune diabetes ${ }^{34}$. In addition, studies have found that $L$. casei could inhibit the alloxan-induced disappearance of pancreatic B cells in mice ${ }^{35}$, which can also inhibit the autoimmune destruction of pancreatic B cells in non-obese diabetic mice ${ }^{36}$. similarly, Lactobacillus GG feeding delays the development of glucose intolerance and hyperglycaemia in streptozotocin-induced diabetic rats ${ }^{37}$, and a significant anti-diabetic effect of a probiotic containing Lactobacillus acidophilus and L. casei has been observed in high-fructose fed rats ${ }^{38}$. Additionally, some probiotics may also induce defensins in epithelial cells, which are natural antimicrobial peptides secreted in the intestine ${ }^{39 .}$

Prebiotics are a group of non-digestible food ingredients including inulin, oligosaccharides, lactulose and resistant starch that are fermented by colonic commensal micro- biota to potentially improve host health by selectively stimulating the growth of certain gut bacteria ${ }^{40}$. Although their targets are traditionally believed to be Bifidobacterium spp. and Lactobacillus spp., prebiotic ingredients may work through the selective stimulation of various indigenous beneficial strains to exert antimicrobial effects, or to modulate immune responses of the host and compete with pathogens for receptors. 

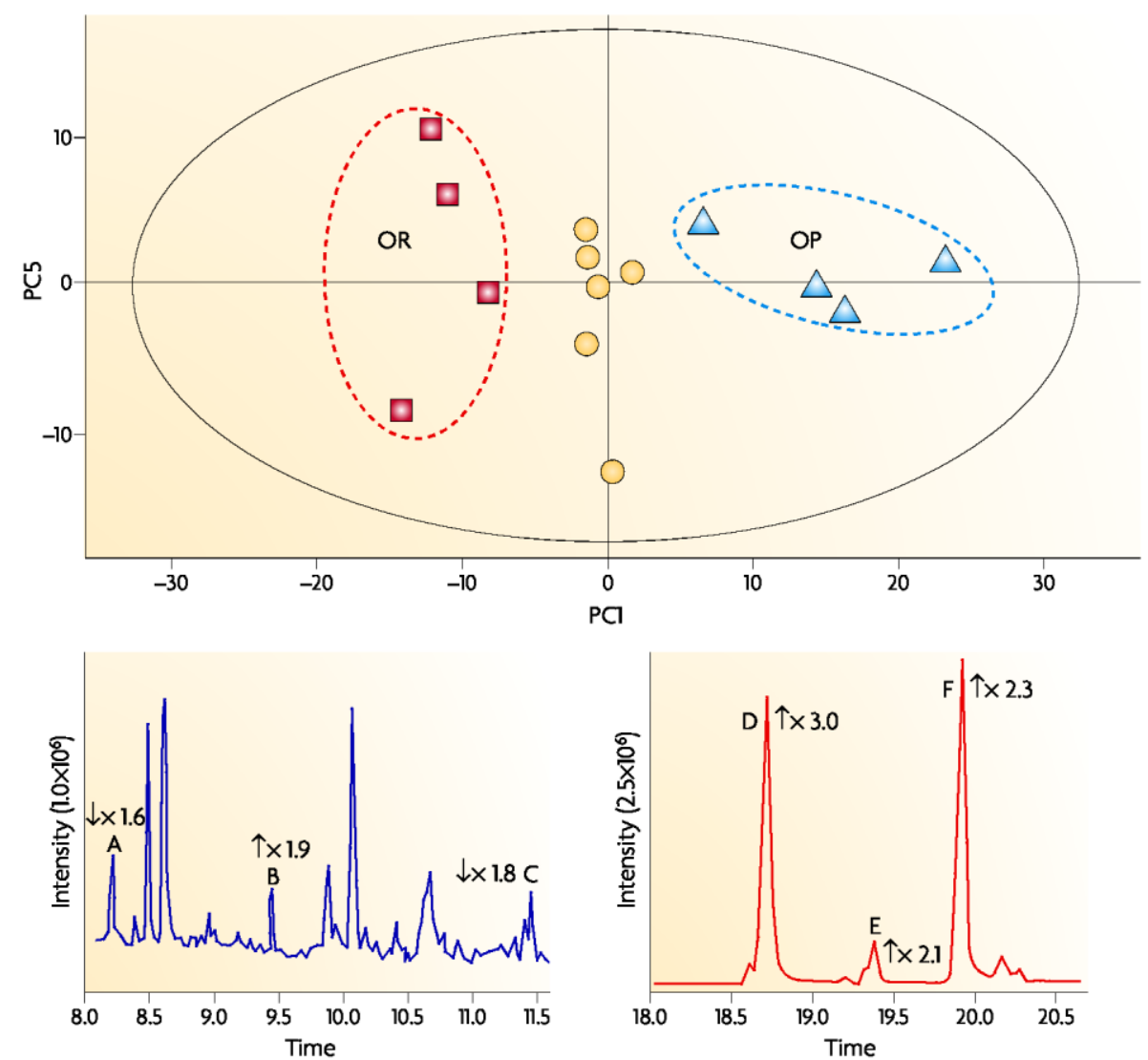

Figure 3 | Metabolic profiling and urinary mammal-microbial metabolites in rats. Pattern recognition (principal components analysis; PCA) of gas chromatography-mass spectrometry (GC-MS) data from urine samples of male Sprague-Dawley rats before exposure to a high-fat diet. The mapping position of the 14 rats is shown with two significant subsets, obesity-prone (OP) and obesity-resistant $(O R)$ rats, which are defined according to their body weight phenotypes after 6 weeks of being fed a high-fat diet, retrospectively. The differential mammal-microbial metabolites contributing to the phenotype development are identified with a supervised method, orthogonal projection to latent structures-discriminant analysis (O-PLS-DA). Full details of this study are reported in REF. 51. A, phenylacetate; B, p-cresol; C, 4-ethyl-phenol; D, hippurate; E, 4-hydroxy-phenylacetate; F, N-phenylacetyl-glycine.

both prebiotics and probiotics have been shown to decrease the incidence and severity of infantile diarrhoea, and prevent antibiotic-induced diarrhoea or food allergies ${ }^{41}$. Additionally, dietary intervention using a combination of probiotics and prebiotics, known as synbiotics, is a feasible treatment strategy and has proven to be efficacious in volunteer trials in patients with active ulcerative colitis ${ }^{42}$. Examples of gut microbiota-related diseases and therapeutic strategies are provided in TABLE 1.

The fact that a significant portion of bioactive metabolites produced in animals and medicinal plants are found to be synthesized or co-synthesized by their commensal microbiota suggests that the gut microbiota itself may be a largely untapped source of novel drugs or drug leads ${ }^{43}$. several anticancer metabolites from marine sponges, including discodermolide, halichondrin $b$ and bryostatin 1, have progressed to preclinical or clinical-trial phases, and are thought to be products derived from their microbiotic consortia ${ }^{44}$. Therefore, the commensal microbiota can potentially be engineered as a bioreactor using new cloning and biosynthetic expression strategies for the controlled secretion of biologically active molecules and vaccines. A new class of 
pharmaceutical compounds derived from the uncultured commensal microorganisms in appropriate in vitro or animal models may be produced to develop a long-term host immunotherapy for diseases such as inflammatory bowel disease. Currently, the application of culture-independent metagenomic technology sheds light on the opportunities of drug discovery from the gut microbiota. The integration of high-throughput DNA sequencing and bioinformatics tools enables the identification of genes encoding bioactive compounds from the commensal microbiota. The genes of interest, isolated directly from commensal bacteria or screened from a clone library using genome-sequence tags, could be cloned into optimal vectors and transformed into surrogate hosts to encode biosynthesis of novel therapeutic products.

\section{Platform technologies for the microbiota}

The development and utilization of appropriate technologies that allow comprehensive and robust analyses for various microorganisms, most of which are non-culturable, are crucial for understanding the impact of the gut microbiota on the host. The established metabolic profiling approach has a powerful capacity for detecting various metabolites originating from microorganisms that are commonly found in mammals. several analytical techniques, including high resolution nuclear magnetic resonance (NMR) spectroscopy ${ }^{45}$ and various gaslliquid chromatography-mass spectrometry (GC-Ms, LC-Ms) techniques ${ }^{46,47}$ are currently used to generate spectral profiles from which information pertaining to pathophysiology can be extracted. for example, the aromatic region of a typical ${ }^{1} \mathrm{H}$ NMR spectrum of urine provides a clear window for visualization of metabolic signature of microbial products, dietary metabolites or parasiterelated metabolites ${ }^{48,49}$. On the basis of a metabolic profiling approach, the concept of pharmacometabonomics, proposed by Clayton et al. ${ }^{50}$ in 2006, highlights the application of such technology to personalized treatment, in which drug-induced responses in individuals can be predicted from a pre-dose metabolic signature. This concept was demonstrated in two classic rat models of dietary-induced obesity and streptozotocin-induced diabetes, in which only certain animals acquired obese or hyperglycaemic phenotypes ${ }^{51}$.

The different outcomes of streptozotocin or dietary intervention were closely associated with variations in pre-dose urinary metabolites that had been reported to originate from gut microbiota metabolism ${ }^{52}$, therefore linking the phenotypic variations to the different pre-dose compositional structures of the gut microbiota (Fig. 3.)

Metagenomics, also known as community genomics, provide an insight into the genetic potential of complex microbial communities, including uncultured species, using culture-independent approaches $^{53}$. It has been used in digestive ecosystems to discover novel hydrolase genes in uncultured rumen bacteria and P-glucanase genes affiliated with uncultured microbiota that colonized the large bowel of mice. Currently, screening approaches for intestinal metagenomic libraries have not been fully established. With the progress in molecular technologies, the most comprehensive 16s ribosomal DNA (rDNA) sequence-based metagenomic approach has been successfully applied in the analysis of the distal gut and faecal micro-biota. This analysis revealed significant differences in community membership between healthy adults ${ }^{54}$, which may contribute to variations in physiology between individuals or in factors that predispose to disease. 
Transplantation of human gut microbiota into surrogate hosts, such as mice or rats, may allow the generation of animal models that mimic human flora and facilitate the in-depth research on human gut microbiota-associated metabolism, pharmacology and immunity ${ }^{55}$. However, the intrinsic differences between rodents and humans in anatomy and physiology make it difficult to directly extrapolate the results. A new human flora-associated piglet model was recently established by the transplantation of human GI microbiota to germ-free piglets with minimal individual variation and ageing patterns that are similar to those observed in humans ${ }^{56}$. such a human flora-mimicking piglet model provides a new platform for pharmacological and biochemical research for gut pathology, nutraceutical and pharmaceutical compound screening, and drug metabolism.

\section{Concluding thoughts: a systems approach}

Gut microbiota-oriented pharmaceutical research requires a progressive, experimental pipeline approach that is dedicated to such a process. Understanding the global relationships between the gut microbiota and humans - for example, a common aetiology for both GI and neurologicallmetabolic disorders - is the first step towards establishing novel diagnostic, therapeutic and preventive modalities for such diseases. strategies that are guided by systems thinking need to be adopted in order to understand the essential elements that are involved in the onset and development of many complex human diseases that are associated with variations in the gut microbiota. focusing on the totality of the microbiota and, therefore, capturing the global change of the gut microbiota with pathological and pharmacological significance is the key to generating comprehensive molecular descriptions of the disease and multifaceted, system-wide drug responses, so that the breadth of biochemical changes contributing to a disease or drug response can be taken into account. research programmes focusing on certain clinical manifestations or phenotypic variations of a disease associated with altered gut microbiota need to be conducted to classify the disease so that different therapeutic strategies can be implemented for different subtypes or states of gut ecology associated with different structures of the microbiota. bioanalytical profiling technologies, such as metabonomics and metagenomics, of patient urinary and faecal samples can be utilized to provide holistic and dynamic biochemical information to assist the medical staff on disease diagnosis, stratification and personalized gut microbiota-targeted treatment. Meanwhile, elucidating the molecular details of host-flora interactions during a pathological process should be pursued to obtain a mechanistic understanding to aid drug development. such a top-down strategy is crucial to achieving a complete coverage of the biochemical mechanisms contributing to the disease to generate feasible treatment measures.

So far, engineering ecologies (the living environments outside and inside of our bodies) has not been that successful because of an incomplete understanding of the systems involved. Currently, we still have limited mechanistic knowledge regarding how indigenous microorganisms have shaped our genome and biology in postnatal development. Emerging technologies, such as metabonomics and metagenomics, have provided information indicating that symbiotic microbes affect metabolic phenotypes and enzyme induction states, which ultimately affect the outcome of interventions ${ }^{4}$. Growing evidence continues to reveal important functions of the gut microbiota in human health and provides new therapeutic targets for the drug industry that, so far, has been focusing exclusively on the human genome. We assert that the value of gut microbiota-targeted drug discovery and therapies can only be fully realized by a major shift from studying the parts 
of living systems in isolation, or the reductionist approach, to studying the intricate interrelationship within our superorganism by integrating results from different scientific disciplines - the systems approach. The host-flora interaction must be composed of complex, networked communications involving convergent, divergent and redundant signalling pathways. Considering the infrastructures for platform technologies to be established and the need to attract cross-disciplinary research personnel, a long-term agenda and research strategy, perhaps 15-20 years in duration, is required for the full development of this field. With the progress of metagenomics in conjunction with other 'omics' technologies, the composition and specific biological functions of the human gut microbiota will be gradually uncovered, opening up an entirely new approach to drug discovery and therapy.

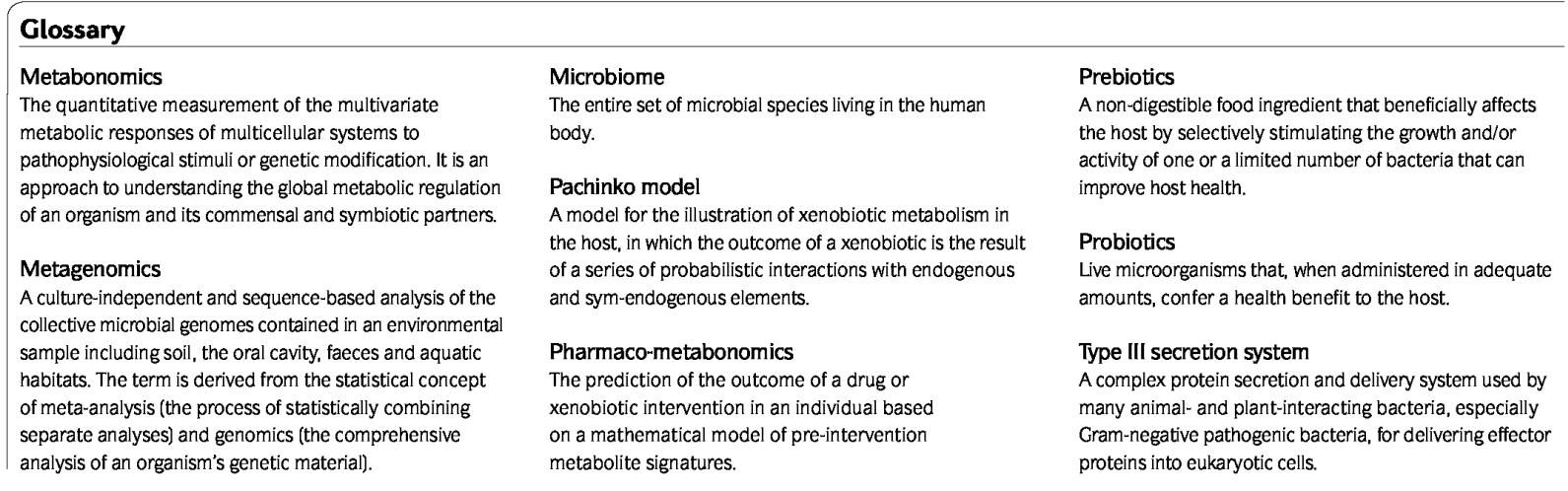


1. Nicholson, J. K., Holmes, E., Lindon, J. C. \& Wilson, I. D. The challenges of modeling mammalian biocomplexity. Nature Biotech. 22, 1268-1274 (2004)

2. Gill, S. R. et al. Metagenomic analysis of the human distal gut microbiome. Science 312, 1355-1359 (2006).

3. Riesenfeld, C. S., Schloss, P. D. \& Handelsman, J. Metagenomics: genomic analysis of microbia communities. Annu. Rev. Genet. 38, 525-552 (2004).

4. Dumas, M. E. et al. Metabolic profiling reveals a contribution of gut microbiota to fatty liver phenotype in insulin-resistant mice. Proc.

5. Turnbaugh, P. J. et al. An obesity-associated gut microbiome with increased capacity for energy harvest Nature 444, 1027-1031 (2006)

6. Ley, R. E., Turnbaugh, P. J., Klein, S. \& Cordon, J. I. Microbial ecology: human gut microbes associated with obesity. Nature 444, 1022-1023 (2006)

7. Brugman, S. et al. Antibiotic treatment partially protects against type 1 diabetes in the Bio-Breeding diabetes-prone rat. Is the gut flora involved in the development of type 1 diabetes? Diabetologia 49 2105-2108 (2006)

8. Strober, W., Fuss, I. \& Mannon, P. The fundamenta basis of inflammatory bowel disease. $J$. Clin. Invest. 117, 514-521 (2007).

9. Chu, F. F. et al. Bacteria-induced intestinal cancer in mice with disrupted $G p \times 1$ and $G p \times 2$ genes. Cancer Res. 64, 962-968 (2004).

10. Guarner, F. \& Malagelada, J. R. Gut flora in health and disease. Lancet 361, 512-519 (2003)

11. Xu, J. \& Gordon, J. I. Honor thy symbionts. Proc. Nat Acad. Sci. USA 100, 10452-10459 (2003)

12. O'Hara, A. M. \& Shanahan, F. The gut flora as a forgotten organ. EMBO Rep. 7, 688-693 (2006)

13. Nicholson, J. K. A Wison, I. D. Understanding 'global' systems biology: metabonomics and the continuum of Drug Discov. 2, 668-676 (2003)

14. Grangette, $C$. et al. Enhanced antiinflammatory capacity of a Lactobacillus plantarum mutant synthesizing modified teichoic acids. Proc. Natl Acad. Sci. USA 102 10321-10326 (2005)

15. Wakabayashi, C., Hasegawa, H., Murata, J. \& Saiki, I. In vivo antimetastatic action of ginseng protopanaxadiol saponins is based on their intestina bacterial metabolites after oral administration. Oncol. Res. 9, 411-417 (1997).

16. Ebbels, T. et al. Toxicity classification from metabonomic data using a density superposition approach: 'CLOUDS' Anal. Chim. Acta 490, 109-122 (2003).

17. Backhed, F. et al. The gut microbiota as an environmental factor that regulates fat storage.
Proc. Natl Acad. Sci. USA 101, 15718-15723 (2004)

18. Yamanaka, K Ohba, H., Hasegawa, A., Sawamura, R. \& Okada, S. Mutagenicity of dimethylated metabolites of inorganic arsenics. Chem. Pharm. Bull. (Tokyo) 37. 2753-2756 (1989).

19. Rizzo, R., Gulisano, M., Pavone, P., Fogliani, F. \& Robertson, M. M. Increased antistreptococcal antibody titers and anti-basal ganglia antibodies in patients with Tourette syndrome: controlled cross-sectional with Tourette syndrome: controlled cross-section
study. J. Child. Neurol. 21, 747-753 (2006).

20. Mell, L. K., Davis, R. L. \& Owens, D. Association between streptococcal infection and obsessivecompulsive disorder, Tourette's syndrome,

21. Nicholson, J. K., Holmes, E. \& Wilson, I. D. Gut microorganisms, mammalian metabolism and personalize

health care. Nature Rev. Microbiol. 3, 431-438 (2005)

22. Arvola, T. et al. Prophylactic Lactobacillus GG reduriaticassod diarrhea in children with antibiotic-associated diarrhea in children with respiratory infections

23. Mendall, M. A. \& Kumar, D. Antibiotic use, childhood affluence and irritable bowel syndrome (IBS). Eur. J. Gastroenterol. Hepatol. 10, 59-62 (1998).

24. Wilcox, M. H. Gastrointestinal disorders and the critically ill. Clostridium difficile infection and pseudomembranous colitis. Best Pract. Res. Clin. Gastroenterol. 17, 475-493 (2003).

25. Jernberg, C., Lofmark, S., Edlund, C. \& Jansson, J.K. Long-term ecological impacts of antibiotic administration on the human intestinal microbiota. ISMEJ. 1, 56-66 (2007).

26. Hadley, C. The infection connection. Helicobacter pylori is more than just the cause of gastric ulcers it offers an unprecedented opportunity to study man microecology and the chronic disease. EMBO Rep. 7, 470-473 (2006)
27. $\mathrm{Xu}, \mathrm{J}$. et al. A genomic view of the human-Bacteroides thetaiotaomicron symbiosis. Science 299, 2074-2076 (2003).

28. Kelly, D. et al. Commensal anaerobic gut bacteria attenuate inflammation by regulating nuclearctoplasmic shuttling of PPAR- $\gamma$ and RelA. Nature Immunol. 5, 104-112 (2004).

29. Samuel, B. S. et al. Genomic and metabolic adaptations of Methanobrevibacter smithii to the human gut Proc. Natl Acad. Sci. USA 104, 10643-10648 (2007). 0. Tampakaki, A. P., Fadouloglou, V. E., Gazi, A. D., Panopoulos, N. J. \& Kokkinidls, M. Conserved features of type III secretion. Cell. Microbiol, 6, 805-816 (2004).

31. Chan, F. K. et al. Eradication of Helicobacter pylori and risk of peptic ulcers in patients starting long-term treatment with non-steroidal anti-inflammatory drugs: a randomised trial. Lancet 359, 9-13 (2002).

32. Rachmilewitz, D. et al. Toll-like receptor 9 signaling mediates the anti-inflammatory effects of probiotics in murine experimental colitis. Gastroenterology 126 , 520-528 (2004).

33. Rastall, R. A. et al. Modulation of the microbial ecology of the human colon by probiotics, prebiotics and synbiotics to enhance human health: an overview of enabling science and potential applications. FEMS Microbiol. Ecol. 52, 145-152 (2005).

34. Matsuzaki, T., Yamazaki, R., Hashimoto, S. \& Yokokura T. Antidiabetic effects of an oral administration of Lactobacillus casei in a non-insulin-dependent diabetes mellitus (NIDDM) model using KK-Ay mice. Endocr. J. 44, 357-365 (1997)

35. Matsuzaki, T. et al. Prevention of onset in an insulin dependent diabetes mellitus model, NOD mice, by ora feeding of Lactobacillus casei. APMIS 105, 643-649 (1997).

36. Matsuzaki, T. et al. Effect of oral administration of actobacillus casei on alloxan-induced diabetes in mice. APMIS 105, 637-642 (1997).

37. Tabuchi, M. et al. Antidiabetic effect of Lactobacillus GG in streptozotocin-induced diabetic rats. Bios Biotechnol. Biochem. 67, 1421-1424 (2003).

38. Yadav, H., Jain, S. \& Sinha, P. R. Antidiabetic effect of probiotic dahi containing Lactobacillus acidophilus and Lactobacillus case in high fructose fed rats. Nutrition 23, 62-68 (2007).

39. Schlee, M. et al. Induction of human $\beta$-defensin 2 by the probiotic Escherichia coll Nissle 1917 is mediated through

40. Gibson, G. R. \& Roberfroid, M. B. Dietary modulation of the human colonic microbiota: introducing the concept of prebiotics. J. Nutr. 125, 1401-1412 (1995).

41. Chen, C. C. \& Walker, W. A. Probiotics and prebiotics: mole in clinical disease states. Adv. Pediatr. 52, 77-113 (2005).

42. Furrie, E. et al. Synbiotic therapy (Bifidobacterium longum/Synergy 1) initiates resolution of inflammation in patients with active ulcerative colitis: a randomised controlled pilot trial. Gut 54, 242-249 (2005).

43. associated microorganisms: distribution, structural diversity, bioactivity, and implications of their occurrence J. Nat. Prod. 69, 509-526 (2006).

44. Dunlap, W. C. et al. Biomedicinals from the phytosymbionts of marine invertebrates: a molecular approach. Methods 42, 358-376 (2007).

45. Brindle, J. T. et al. Rapid and noninvasive diagnosis of the presence and severity of coronary heart disease 1439-1444 (2002)

46. Oiu, Y. et al. Application of ethyl chloroformate derivatization for gas chromatography-mass pectrometry based metabonomic profiling. Anal. Chim. Acta 583, 277-283 (2007).

47. Wang, X. et al. Simultaneous determination of 17 ginsenosides in rat urine by ultra performance liquid chromatography-mass spectrometry with solid-phase extraction. Anal. Chim. Acta 594, 265-273 (2007).

48. Phipps, A. N., Stewart, J., Wright, B. \& Wilson, I. D. Effect of diet on the urinary excretion of hippuric acid and other dietary-derived aromatics in rat. A complex interaction between diet gut microflora and substrate specificity. Xenobiotica 28, 527-537 (1998).

49. Wang, Y. et al. Metabonomic investigations in mice infected with Schistosoma mansoni: an approach for biomarker identification. Proc. Natl Acad. Sci. USA 101, 12676-12681 (2004).

50. Clayton, T. A. et al. Pharmaco-metabonomic phenotyping and personalized drug treatment. Nature 440, 1073-1077 (2006).
51. Li, H. et al. Pharmacometabonomic phenotyping reveals different responses to xenobiotic interventio in rats. J. Proteome Res. 6, 1364-1370 (2007).

52. Williams, R. E., Eyton-Jones, H. W., Farnworth, M. J. Gallagher, R. \& Provan, W. M. Effect of intestinal on the urinary metabolic profile of a ' $\mathrm{H}$-nuclear magnetic resonance spectroscopy study. Xenobiotica 32, 783-794 (2002).

53. Handelsman, J. Metagenomics: application of genomics to uncultured microorganisms. Microbiol. Mol. Biol. Rev. 68, 669-685 (2004)

54. Eckburg, P. B. et al. Diversity of the human intestinal microbial flora. Science 308, 1635-1638 (2005)

55. Wilcks, A., van Hoek, A. H., Joosten, R. G., Jacobsen, B. B. \& Aarts, H. J. Persistence of DNA studied in different $e x$ vivo and $i n$ vivo rat models simulating the human gut situation. Food Chem. Toxicol. 42, 493-502 (2004).

56. Pang, $X$, et al. Inter-species transplantation of gut microbiota from human to pigs ISMEJ. 1, 156-162 (2007).

57. Malfertheiner, P. et al. Helicobacter pylori eradication and gastric ulcer healing - comparison of three pantoprazole-based triple therapies. Aliment Pharmacol. Ther. 17, 1125-1135 (2003).

58. Surawicz, C. M. et al. Prevention of antibioticassociated diarrhea by Saccharomyces boulardii: a prospective study. Gastroenterology $96,981-988$ (1989).

59. Surawicz, C. M. et al. The search for a better treatment for recurrent Clostridium difficile disease: use of highdose vancomycin combined with Saccharomyces Dis. 31, 1012-1017 (2000).

60. Cummings, J. H. Macfarlane, G. T. \& Macfarlane S. Intestinal bacteria and ulcerative colitis. Curr. Issues Intest. Microbiol. 4, 9-20 (2003).

61. Subramanian, S., Campbell, B. J. \& Rhodes, J. M. Bacteria in the pathogenesis of inflammatory bowel
disease. Curr. Opin. Infect. Dis. 19, 475-484 (2006).

62. Andoh, A.\& Fujiyama, Y. Therapeutic approaches targeting intestinal microflora in inflammatory bow disease. World J. Gastroenterol. 12, 4452-4460 (2006)

63. O'Mahony, L. et al. Probiotic impact on microbia flora, inflammation and tumour development in IL-10 knockout mice. Aliment. Pharmacol. Ther. 15 1219-1225 (2001)

64. Singh, J. et al. Bifidobacterium longum, a lactic acidproducing intestinal bacterium inhibits colon cancer and modulates the intermediate biomarkers of colon carcinogenesis. Carcinogenesis 18, 833-841 (1997).

65. Dobbs, R, J. et al. Role of chronic infection and inflammation in the gastrointestinal tract in the etiology and pathogenesis of idiopathic parkinsonism. Part 1: eradication of Helicobacter in the cachexia of idiopathic parkinsonism. Helicobacter 10, 267-275 (2005).

66. Bjarnason, I. T. et al. Role of chronic infection and inflammation in the gastrointestinal tract in the etiology and pathogenesis of idiopathic parkinsonism. Part 2. response of facets of clinical idiopathic parkinsonism to Helicobacter pulori eradication. A randomized, double-blind, placebo-controlled efficacy study. Helicobacter 10, 276-287 (2005)

67. Weller $\mathrm{C}$. et al Role of chronic infection and inflammation in the gastrointestinal tract in the etiolog and pathogenesis of idiopathic parkinsonism. Part 3 predicted probability and gradients of severity of idiopathic parkinsonism based on $\mathrm{H}$. pylori antibody profile. Helicobacter 10, 288-297 (2005).

\section{Acknowledgements}

This work was financially supported by the National Basic Research Program of China (2007CB914700) and the International Collaborative Project, Chinese Ministry of Science and Technology (2006DFA02700).

DATABASES

Entrez Genome Project: http://www.ncbi.nlm,nih.gov/ entrez/query.fcgi?d $b=g$ enomepri

Bacteroides thetaiotaomicron | Clostridium difficile | Escherichia coli| Helicobacter pylori| Lactobacillus acidophilus |Lactobacillus casei $\mid$ Methanobrevibacter smithii

http://wnww.ncbi.nlm.nih.gov/entrez/query.fcgi?db=OMIM ADHD | inflammatory bowel disease | obesity |

Tourette syndrome

FURTHER INFORMATION

The Consortium on Metabonomic Toxicology

htp://bc-comet.sk,med,ic,ac,uk/

ALL LINKS ARE ACTIVE IN THE ONLINE PDF 